\title{
Bounce effect seen in opposite motions
}

\author{
SHIGEMASA SUMI
}

Psychology Laboraton, Keio University, Hivoshi, Kohoku-ku, Yokohama 223

Kanizsa's (1979) ostrich appeared as if he were dancing in a very curious fashion. His legs actually passed one another but they seemed to be bouncing back to their original end point. The bounce effect appeared also in the opposite motions of rotating disks. At the meeting on a circular path, they were not seen passing one another but bouncing off; one of them was seen remaining throughout on one side and the other remaining throughout on the opposite side. The smaller the difference between the speeds of colliding objects, the more often the bounce effect was seen. When the moving disks were moved at very high speeds, they always showed the bounce effect at each meeting. The bounce effect was more frequently perceived in binocular vision than in monocular. and in the brightly lit surroundings than in the dark ones. The factors of symmetry and smoothness determining the bounce effect seen in opposite motions were discussed.

Key words: Gestalt factor, motion perception, kinetic occlusion, symmetry, smooth pursuit.

Kanizsa (1979) produced a model of a little man. The two arms and the two legs of the model were made to move simultaneously oscillating in the direction opposite to each other, the one passing in front of the other. When the apparatus was set in motion, the little man was expected to walk, but he did not do so. He was seen to be hopping about as if he were doing comical gymnastics. His legs actually passed one another, as shown in Figure 1A, but appeared to be bouncing off to their original end point at the moment of contact as in Figure 1B. One leg of the little man was seen remaining throughout on one side and the other leg remaining throughout on the other side. I found this kind of phenomenon appearing in the two objects moving in the direction opposite to each other. As I call it 'bounce effect', so the name is used here. Kanizsa produced another model of an ostrich and observed also the bounce effect in the movements of the bird' legs. The ostrich's legs actually passed one another but they were seen bouncing so that the

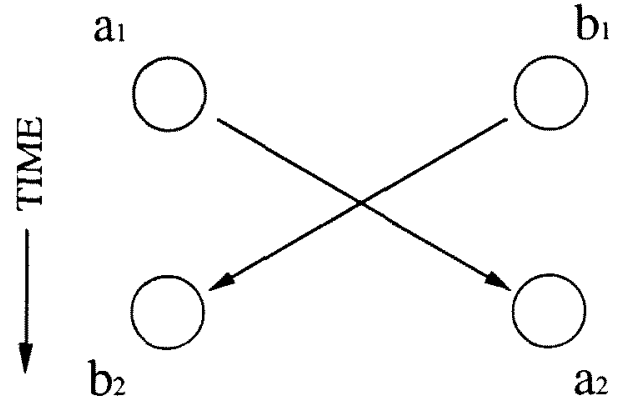

PASSING THROUGH $\mathrm{a}_{1}$

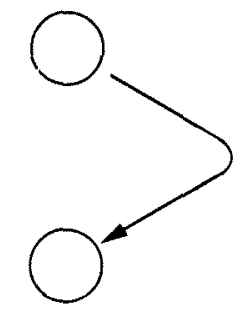

a2

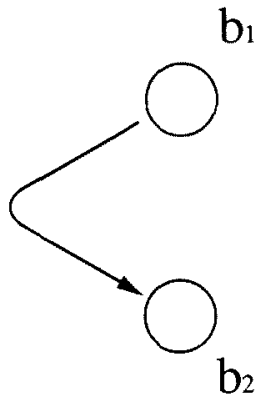

$\mathrm{b}_{2}$

\section{BOUNCING OFF}

\section{A}

B

Figure 1. In the Kanizsa little man, his legs changed the position from $a_{1}$ to $a_{2}$ and from $b_{1}$ to $b_{2}$ ( $A$ ). But they appeared, at the moment of contact, to bounce off to their original end points ( $B$ ). 
bird looked as if he were dancing in a very curious fashion.

The fact previously stated by Katz (1950) is very interesting. He took the motion pictures of a walking person. Superimposing the mirror image of the original scene on the original one, he produced the double-exposure film. In the film the two images of the same person simultaneously walking from left to right and from right to left was shown. At the moment of contact, the person appeared passing through himself as if he were made of 'air', and then separating again. This mirror image effect, so termed by Katz, points out an important aspect of the bounce effect. Strictly speaking, 'passing through' means the perceptual processes in which one of two moving objects is seen going behind another and coming from behind it, and it never means that either is going out of existence. Therefore there is a close connection between the passing through and the occluding the surface edges. These two perceptual processes seem to be mutually dependent. If the kinetic occluding does not occur, the passing through also will not be perceived and it will be replaced with the bouncing off. Passing through is intrinsically three-dimensional and it is caused by two motions appearing on the overlapped surfaces. On the other hand the bouncing off is intrinsically two-dimensional and it is caused by two motions appearing on the same plane. When neither passing nor bouncing is seen, such an ambiguous pattern of the mirror image effect by Katz will be experienced.

Kanizsa (1979) pointed out that such variables as frequency and amplitude of oscillation, coloration, illumination, and fixation point of visual attention would be involved in the bounce effect. My preliminary observations suggested that those factors of motion speed, size and brightness of the moving stimuli and visual regard would be more influential upon the bounce effect (Sumi, 1988). In the present study it was attempted to examine in detail the effects of both motion speed and viewing condition on the bounce effect.

Method

\section{Stimuli}

Two small disks, each $8 \mathrm{~cm}$ in diameter, were made from white cardboard, attached to both ends of a thin steel-stick $(52 \mathrm{~cm}$ in length). The disk's center was placed to be identical with the end of the stick. The midpoint of the stick was fixed on the coaxial pivot, driven by an electrically controlled pulse motor so as to rotate in the clockwise (CW) direction. The stick and the disks (CW-disks) rotated together around the coaxial pivot. The two disks moved on the circular path, $52 \mathrm{~cm}$ in diameter. The second stick was fixed on the coaxial pivot, driven also by the second electric pulse motor so as to rotate in the counterclockwise ( $\mathrm{CCW}$ ) direction. The stick and the disks (CCW-disks) also rotated together around the coaxial pivot. When the apparatus was set in motion, both of the $\mathrm{CW}$-disks and $\mathrm{CCW}$-disks moved simultaneously on the same circular path in the direction opposite to each other (Figure 2A). When those disks met each other at any point on the circular path, they overlapped and passed through with the distance of 1.0 $\mathrm{cm}$ or $1.5 \mathrm{~cm}$ in depth. One of two gray boards was placed behind the moving disks and the other in front of them. The former served as the background and the latter screened the rotating sticks. The second gray board placed in front had the shape of a semicircle so that the moving disks could be seen to move along the circular edge of it (Figure $2 B$ ). At the center of the semicircular board a small white spot $(0.5 \mathrm{~cm}$ in diameter $)$ was placed, used as the fixation spot. The moving disks were illuminated by the tungsten 
lamps from the top, left and right, with a mean luminance of $27.9 \mathrm{~cd} / \mathrm{m}^{2}$.

\section{Procedure}

The CW-disks' motion (CW-motion) was presented at five different speeds: 150, 200 , 250,300 and $350 \mathrm{~cm} / \mathrm{s}$. On the other hand, the $\mathrm{CCW}$-disks motion (CCW-motion) was presented at thirteen different speeds, that is. from 60 to $300 \mathrm{~cm} / \mathrm{s}$ in steps of $20 \mathrm{~cm} / \mathrm{s}$. As the position of observer's eye (s) was placed at the distance of $280 \mathrm{~cm}$ from the center of rotation (the coaxial pivot), the speed of the moving disks ranged from 12.3 to 71.6 degree (in visual angle)/s.

The subject sat in a chair with his head on the chin rest. The position of his eye (s) was adjusted so as to be placed at the same height as the fixation spot, indicated as " $F$ " in Figure 2B. The fixation spot, white spot, was made by a card $(0.5 \mathrm{~cm}$ in diameter), placed on the position which was identical with the center of rotation of the moving disks. To control the effect of eye-movements, the subject was asked to fix his eye (s) on the fixation spot. When the fixation spot was gazed at accurately, the moving disks appeared in the periphery of the visual field, at about $5.3^{\circ}$ from the center.

The moving disks were viewed; (1) in the brightly lit surroundings with both eyes (L-B condition), (2) in the brightly lit surroundings with one eye (L-M condition), and (3) in the dark surroundings with one eye (D-M condition). In the last view-condition, the moving disks were viewed through a tube so that the visual field was limited to the extension of $30^{\circ}$ while the other eye was screened. The subjects observed the three view-conditions in the order of L-B, L-M, and D-M, but no subject observed more than one condition on one day. In each view-condition, the sixtyfive different motion-conditions (=five $\mathrm{CW}$ motions $\times$ thirteen $\mathrm{CCW}$-motions) were presented to the subjects. For each $\mathrm{CW}$-motion, the speed of CCW-motion increased (an

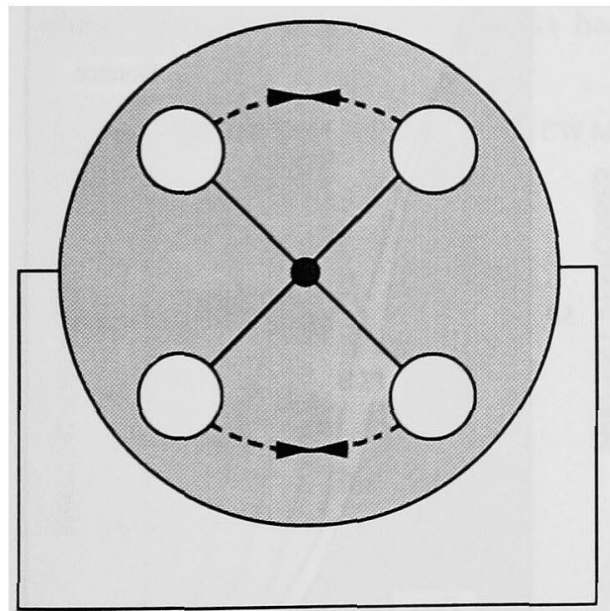

A

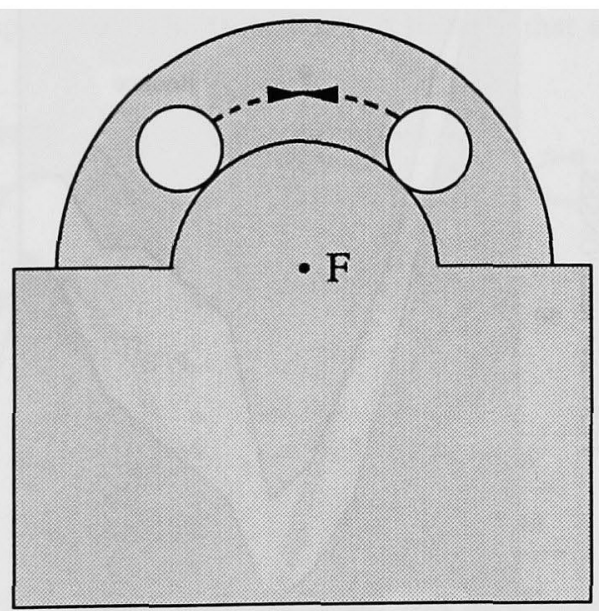

B

Figure 2. Four disks, two each attached on the ends of a stick and rotated around the midpoint of the stick, moved in the direction opposite to each other on the same circular path (A). Paying attention to the fixation point $(F)$, subjects observed the moving disks appearing from the left to the right and from the right to the left along the semi-circular edge of the gray board (B). 
ascending trial) and then decreased (a descending trial), step by step. The subjects conducted two ascending and two descending trials in L-B condition, and one ascending and one descending trials in L-M and D-M conditions. Each motion-condition was presented to the subjects for $20 \mathrm{~s}$ to $30 \mathrm{~s}$, during which time they reported verbally how the moving disks were seen. It took forty to seventy minutes for the subjects to make their observations on 65 motions in each viewcondition.

\section{Subjects}

Five to six staff members of psychology laboratory who were not informed about the purpose of the experiment, participated in the experiment.

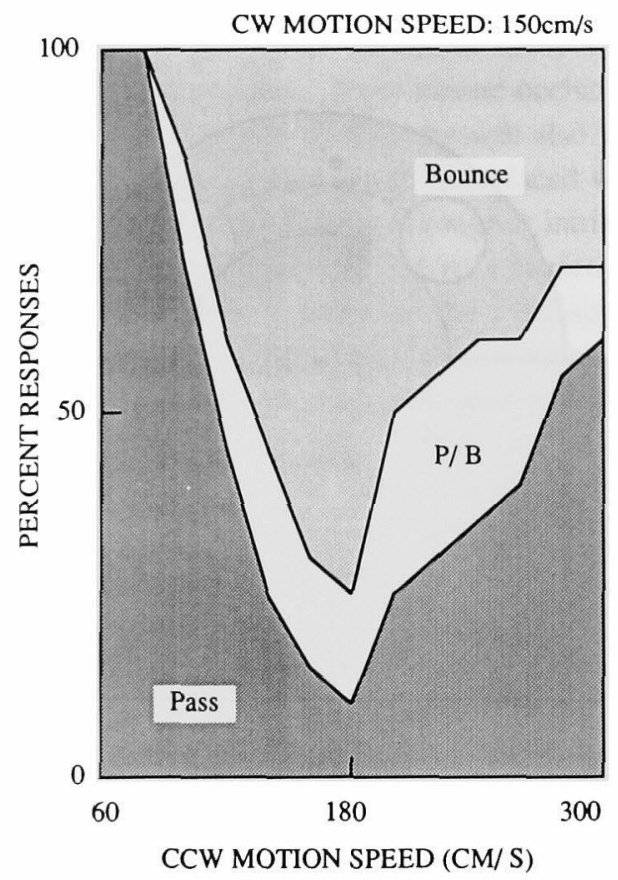

\section{Results}

The responses obtained from the subjects were classified into three categories: (1) "pass" when two moving disks at the contact appeared passing one another; (2) "bounce" (a) when the two moving disks at the contact were seen hitting violently each other and bouncing off, (b) in a collision either was seen to move back quickly as if it were hit by swinging a bat, or (c) either was seen thrown up in the air and rebounding like a ball tossed in volleyball game; and (3) "pass/bounce $(\mathrm{p} / \mathrm{b})$ " when "pass" and "bounce" alternately appeared at the meeting of the moving disks.

The influence of motion speed on the bounce effect was seen as very clear. When

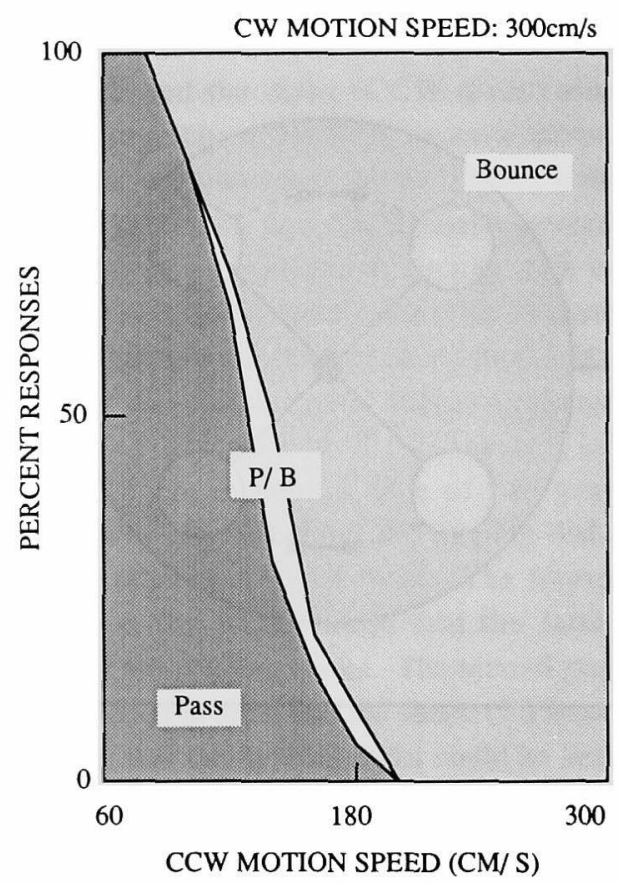

Figure 3. The responses $(N=20)$ obtained from the five subjects under the $\mathrm{L}-\mathrm{B}$ view-condition were divided into three categories of pass, pass/bounce and bounce, and their frequencies against the thirteen CCW-motion speeds were shown for the two different CW-motion speeds. 
the moving disks moved faster or when the speed difference between the $\mathrm{CW}$ - and $\mathrm{CCW}$-disks became smaller, the bounce effect more frequently occurred. In Figure 3 the results in the L-B condition are shown. At the $\mathrm{CW}$-motion of $150 \mathrm{~cm} / \mathrm{s}$, the bounce effect appeared more frequently when the speed difference between $\mathrm{CW}$ - and $\mathrm{CCW}$ motions became smaller. At the $\mathrm{CW}$-motion of $300 \mathrm{~cm} / \mathrm{s}$, on the other hand, when the CCW-disks moved at a speed beyond 180 $\mathrm{cm} / \mathrm{s}$, the bounce effect always appeared in their collision. ANOVA revealed the significant effects of CW-and CCW-motion and of the interaction between them in the L-B condition: $F(4.16)=17.73, p<.01, F(12,48)$ $=188.55, p<.01$, and $F(48,192)=3.35, p$ $<.01$. Similar results were obtained in the L$M$ condition; $F(4,20)=6.21, p<.01, F(12$, $60)=50.62, p<.01, F(48,240)=4.76, p<$ .01 , and also in the D-M condition; $F(4,20)$ $=9.57, p<.01 . F(12,60)=188.55, p<.01$, $F(48,240)=3.35, p<.01$. The influence of view-condition on the bounce effect was shown in Figure 4. In all view-conditions, when the oppositely moving disks had a small difference between their speeds, very frequently the bounce effect appeared. Namely. when the CW-disks moved at a speed of $150 \mathrm{~cm} / \mathrm{s}$ and CCW-disks moved at speeds of 160 to $200 \mathrm{~cm} / \mathrm{s}$, the effect very frequently occurred, $F(12,24)=10.18, p<$ .01 , appearing more in L-B condition than the rest, $F(2,24)=27.05, p<.01$. And also, when the disks were moved at both $200 \mathrm{~cm} / \mathrm{s}$ in $\mathrm{CW}$-motion and $240 \mathrm{~cm} / \mathrm{s}$ in $\mathrm{CCW}$ motion, the effect most often occurred, $F(12$, $24)=29.43, p<.01$, appearing more in the L-B condition than the rest, $F(2,24)=24.79, p$ $<.01$. When they were moved both at 250 $\mathrm{cm} / \mathrm{s}$ in $\mathrm{CW}$-motion and at $200 \mathrm{~cm} / \mathrm{s}$ and more in $\mathrm{CCW}$-motion, the effect very often occurred, $F(12,24)=251.58, p<.01$, appearing in all view-conditions without significant difference between them, $F(2,24)=2.79$.

\section{Discussion}

The results revealed that the smaller the difference between the speeds of colliding objects, the more often the bounce effect appeared. The facts seem to indicate that a

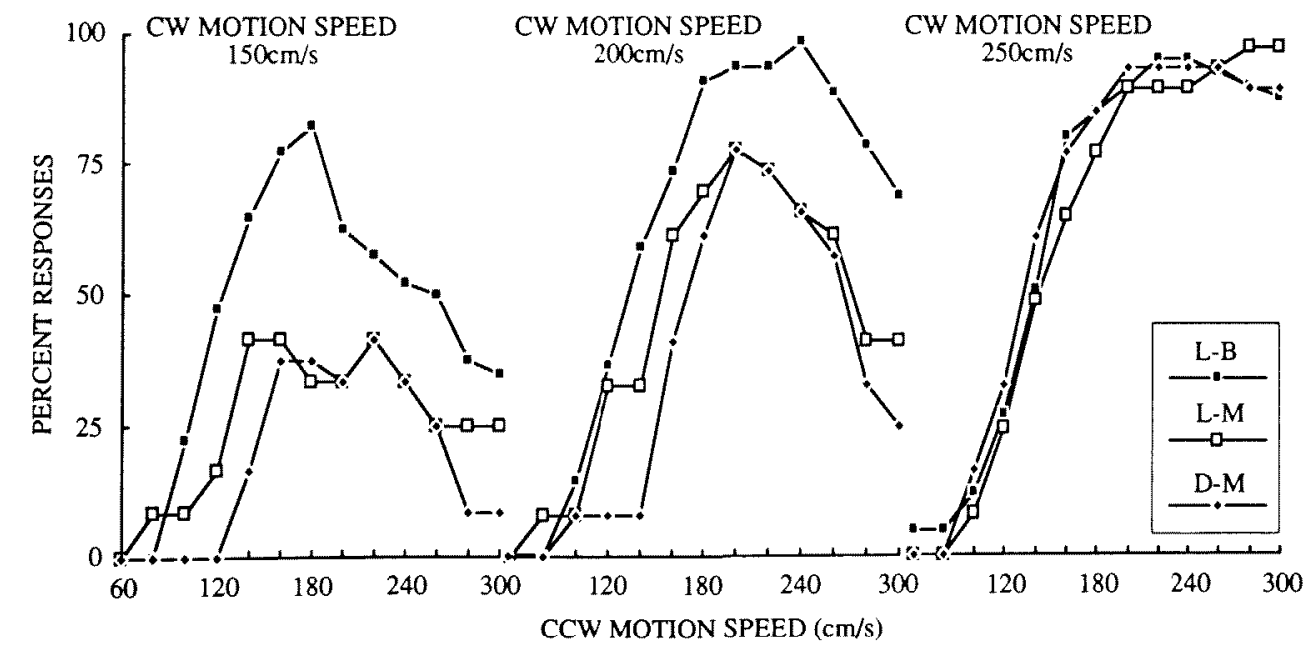

Figure 4. The frequencies of the response judged as "bounce" were plotted against the thirteen CCW-motion speeds. The results obtained in the three different view-conditions and in the three different $\mathrm{CW}$-motion speeds were shown. 
symmetrical arrangement in opposite motions produced more the perception of bounce effect. According to Metzger (1953), when the objects, rotating three-dimensionally in depth, cast their symmetrical shadows onto the screen, their motions were seen expanding and contracting just like an accordion. He called the effect "Ziehharmonikabewegung (accordion-movement)". The bounce effect obtained in the present study can be considered as a kind of "Ziehharmonika-bewegung", giving us the evidence that the factor of symmetry took an important role in the perceptual motion configuration.

The results showed that, the greater the difference between the motion speeds of the moving disks, the more often the passing through appeared. For the occurrence of passing through or for the occurrence of kinetic occlusion, the smoothness of perceived motion seemed to be very important. The factor of smoothness was assumed to be chiefly dependent for its effectiveness on the ocular smooth pursuit. If both the occluding object and the occluded one were simultaneously moved at a very high speed, observers could pursue neither with enough fixation. The lack of smoothness in the moving objects seemed to make it difficult for the observers to perceive the passing through in opposite motions.

Other evidence, given by Sumi (1988), is very suggestive of the present results. He examined how the oppositely moving disks, as used in the present study, would appear under strobe at the flash rates of $8 \mathrm{~Hz}$ or less. In the situation, the moving disks were mostly observed as bouncing off, not as passing through, even though their motion speeds differed considerably. The intermittent lighting by strobe-flashes at a low rate made it difficult for the observers to pursue smoothly either one of the two oppositely moving objects with fixation and it seemed to bring about the loss of smoothness which was the determinant for the perceptual organization of oppositely moving objects. In the lack of the temporal factor of smoothness by the strobe, it was found that the moving objects came to be seen as organized by the spatial factor of proximity or nearness (Sumi, 1988). In the present study, the bounce effect in the opposite motions frequently appeared in their high speed conditions. The facts were then considered as resulting from the lack of perceptual smoothness in the oppositely moving objects.

The bounce effect was seen more often in binocular vision than in monocular, and more often in a brightly lit surround than in a dark one. The results implied that in a brightly lit surround the factor of smoothness would not be so powerfully operative and instead the factor of symmetry would become stronger. Dancing ostrich demonstrated by Kanizsa (1979) let us know thus how important the factor of symmetry will be in the perception of oppositely moving objects and how the extent to which the factor of symmetry will inhibit the perception of the passing through.

\section{References}

Gibson, J.J. 1979 The ecological approach to visual perception. Houghton Mifflin: Boston

Kanizsa, G. 1979 Organization in vision: Essays on Gestalt perception. New York: Praeger.

Katz, D. 1950 Gestalt Psychology: Its nature and significance. (Reprinted in 1979) Westport, CT: Greenwood Press

Metzger, W. 1953 Gesetze des Sehens. Frankfurt: Waldemar Kramer

Sumi, S. 1988 Passing and rebounding in visual objects moving in opposite directions. Perception, 17, 405 (Abstract). (Received Dec. 6, 1994; accepted 21, 1995) 